\title{
Existence and Linear Stability of Equilibrium Points in the Robe's Restricted Three-Body Problem with Oblateness
}

\author{
Jagadish Singh $^{1}$ and Abubakar Umar Sandah ${ }^{2}$ \\ ${ }^{1}$ Department of Mathematics, Faculty of Science, Ahmadu Bello University, Zaria, Nigeria \\ ${ }^{2}$ Department of Mathematics Statistics and Computer Science, College of Science \\ and Technology, Kaduna Polytechnic, Kaduna, Nigeria \\ Correspondence should be addressed to Abubakar Umar Sandah, usandabu@yahoo.com
}

Received 26 March 2012; Accepted 2 July 2012

Academic Editor: Burak Polat

Copyright (C) 2012 J. Singh and A. Umar Sandah. This is an open access article distributed under the Creative Commons Attribution License, which permits unrestricted use, distribution, and reproduction in any medium, provided the original work is properly cited.

This paper investigates the positions and linear stability of an infinitesimal body around the equilibrium points in the framework of the Robe's circular restricted three-body problem, with assumptions that the hydrostatic equilibrium figure of the first primary is an oblate spheroid and the second primary is an oblate body as well. It is found that equilibrium point exists near the centre of the first primary. Further, there can be one more equilibrium point on the line joining the centers of both primaries. Points on the circle within the first primary are also equilibrium points under certain conditions and the existence of two out-of-plane points is also observed. The linear stability of this configuration is examined and it is found that points near the center of the first primary are conditionally stable, while the circular and out of plane equilibrium points are unstable.

\section{Introduction}

Robe [1] considered a new kind of restricted three-body problem in which, one of the primaries of mass $m_{1}$ is a rigid spherical shell, filled with homogenous, incompressible fluid of density $\rho_{1}$; the second one is a point mass $m_{2}$ located outside the shell and moving around the mass $m_{1}$ in a Keplerian orbit; the infinitesimal mass $m_{3}$ is a small sphere of density $\rho_{3}$, moving inside the shell and is subject to the attraction of $m_{2}$ and the buoyancy force due to the fluid of the first primary. Further, he discussed the linear stability of an equilibrium point obtained in two cases. In the first case, the orbit of $m_{2}$ around $m_{1}$ is circular and in the second case, the orbit is elliptic, but the shell is empty (there is no fluid inside it) or densities of $m_{1}$ and $m_{3}$ are equal. Since then various studies (e.g., [2-4]) under different assumptions have been carried out. 


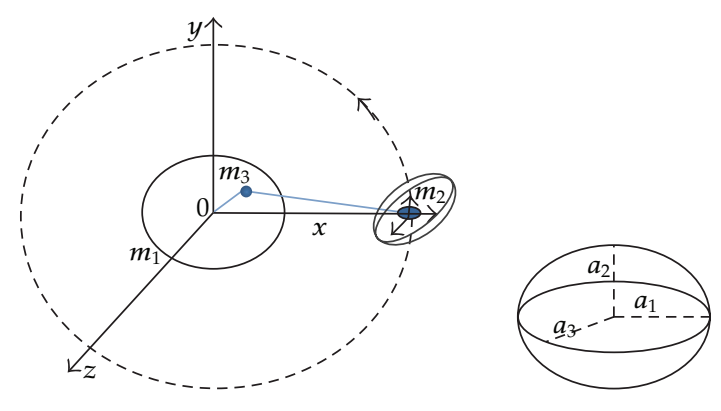

Figure 1: The Robe's CRTBP with oblate primaries.

In his study, Robe [1] assumed that the pressure field of the fluid $\rho_{1}$ has a spherical symmetry around the center of the shell and he took into account only one out of the three components of the pressure field which is due to the own gravitational field of the fluid $\rho_{1}$. He did not consider the other two components arising from the attraction of $m_{2}$ and the centrifugal force. Taking care of all these three components of the pressure field, A. R. Plastino and A. Plastino [5] reanalyzed the Robe's. But in their study, they assumed the hydrostatic equilibrium figure of the first primary as Roche's ellipsoid (see Figure 1). They found that when the density parameter $D$ is taken as zero, every point inside the fluid is an equilibrium point; otherwise the center of the ellipsoid is the only equilibrium point and it is linearly stable.

Hallan and Rana [3] investigated the existence of all equilibrium point and their stability in the Robe's [1] restricted three-body problem. It was seen that the Robe's elliptic restricted three-body problem has only one equilibrium point for all values of the density parameter $K$ and the mass parameter $\mu$, while the Robe's circular restricted three-body problem can have two, three, or infinite numbers of equilibrium points. As regards to the stability of these equilibria, they confirmed the stability result given by Robe [1] of the equilibrium point $(-\mu, 0,0)$, whereas triangular and circular points are always unstable. The equilibrium point collinear with the center of the shell and the second primary was found to be stable under some conditions.

Hallan and Mangang [4] studied the Robe's [1] restricted three-body problem by considering the full buoyancy force as in A. R. Plastino and A. Plastino [5] and assuming the hydrostatic equilibrium figure of the first primary as an oblate spheroid. They derived the pertinent equations of motion and discussed the existence of equilibrium point and their linear stability.

The participating bodies in the classical restricted three-body problem are strictly spherical in shape, but in actual situations several heavenly bodies, such as Saturn and Jupiter, are sufficiently oblate. The minor planets and meteoroids have irregular shape. The lack of sphericity, or the oblateness, of the planet causes large perturbations from a two-body orbit. The motions of artificial Earth satellites are examples of this. Global studies of problems with oblateness have been carried out by many researchers (e.g., [6-9]).

Therefore, our effort in this paper aims at investigating the equilibrium points and their stability in the Robe's circular restricted three-body problem when the hydrostatic equilibrium figure of the fluid of the first primary is an oblate spheroid and the second one is an oblate spheroid as well. The model of this study can be used to study the small oscillation of the Earth's inner core taking into account the Moon's attraction. 
This paper is organized as follows; Section 2 represents the equations of motion; the existence of the equilibrium points is mentioned in Section 3, while Section 4 investigates their linear stability; Section 5 discusses the results obtained; the conclusion is drawn in Section 6.

\section{Equation of Motion}

Let the first primary $m_{1}$ be a fluid of density $\rho_{1}$ in the shape of an oblate spheroid as assumed by Hallan and Mangang [4]; let the second primary $m_{2}$ be an oblate body too as Sharma and Subba Rao [6] assumed, which describes a circular orbit around $m_{1}$.

We adopt a uniformly rotating coordinate system $O x_{1} x_{2} x_{3}$ with origin at the center of mass $m_{1}, O x_{1}$ pointing towards $m_{2}$, with $O x_{1} x_{2}$ being the orbital plane of $m_{2}$ coinciding with the equatorial plane of $m_{1}$. Then, the equations of motion of the infinitesimal body of density $\rho_{3}$ in the coordinate system take the form $[4,6]$ :

$$
\ddot{x}_{1}-2 n \dot{x}_{2}=\frac{\partial U}{\partial x_{1}}, \quad \ddot{x}_{2}+2 n \dot{x}_{1}=\frac{\partial U}{\partial x_{2}}, \quad \ddot{x}_{3}=\frac{\partial U}{\partial x_{3}},
$$

where

$$
\begin{gathered}
U=V+\frac{n^{2}\left\{\left(x_{1}-\left(m_{2} /\left(m_{1}+m_{2}\right)\right) R\right)^{2}+x_{2}^{2}\right\}}{2}, \\
V=B+B^{\prime}-\frac{\rho_{1}}{\rho_{3}}\left[B+B^{\prime}+\frac{n^{2}\left\{\left(x_{1}-\left(m_{2} /\left(m_{1}+m_{2}\right)\right) R\right)^{2}+x_{2}^{2}\right\}}{2}\right], \\
B=\pi G \rho_{1}\left[I-A_{1} x_{1}^{2}-A_{1} x_{2}^{2}-A_{2} x_{3}^{2}\right], \\
B^{\prime}=\frac{G m_{2} \alpha_{2}}{\left[\left(R-x_{1}\right)^{2}+x_{2}^{2}+x_{3}^{2}\right]^{1 / 2}}+\frac{3 G m_{2} \alpha_{2} x_{3}^{2}}{2\left[\left(R-x_{1}\right)^{2}+x_{2}^{2}+x_{3}^{2}\right]^{3 / 2}}-\frac{2\left[\left(R-x_{1}\right)^{2}+x_{2}^{2}+x_{3}^{2}\right]^{5 / 2}}{2}, \\
A_{1}=a_{1}^{2} a_{2} \int_{0}^{\infty} \frac{d u}{\Delta\left(a_{1}^{2}+u\right)}, \\
\Delta^{2}=\left(a_{1}^{2}+u\right)^{2}\left(a_{2}^{2}+u\right), a_{2}^{2} A_{2}, \\
n^{2}=\frac{A_{2}=a_{1}^{2} a_{2} \int_{0}^{\infty} \frac{d u}{\Delta\left(m_{1}+m_{2}\right)}}{R^{2}}\left(1+\frac{3}{2} \alpha_{1}+\frac{3}{2} \alpha_{2}\right) ; \alpha_{1}=\frac{a_{1}^{2}-a_{3}^{2}}{5 R^{2}}, \alpha_{2}=\frac{a_{2}^{2}-a_{4}^{2}}{5 R^{2}}, \\
D=1-\frac{\rho_{1}}{\rho_{3}} .
\end{gathered}
$$


Here $V$ is the potential that explains the combined forces upon the infinitesimal mass, $B$ denotes the potential due to the fluid mass of the first primary, $B^{\prime}$ stands for the potential due to the second primary, $R$ is the distance between the primaries, and $G$ is the gravitational constant. $n$ is the mean motion. $a_{1}, a_{2}$ and $a_{3}, a_{4}$ are the equatorial and polar radii of the first and second primary, respectively. $I$ stands for the polar moment of inertia, while $A_{i}(i=$ $1,2)$ are the index symbols. $\alpha_{1}$ and $\alpha_{2}$ are the oblateness coefficients of the first and second primaries, respectively.

We choose the unit of mass such that the sum of the masses of the primaries is taken as unity, thus we take $m_{2}=\mu, 0<\mu=m_{2} /\left(m_{1}+m_{2}\right)<1$. For the unit of length, we take the distance between the primaries as unity, that is, $R=1$ and the unit of time is also selected such that $G=1$. With these units and substituting the expression for the potential $B$ due to the fluid in the first primary and the potential $B^{\prime}$ due to the second oblate primary, the equations of motion (2.1) are recast to the form:

$$
\ddot{x}_{1}-2 n \dot{x}_{2}=\frac{\partial U}{\partial x_{1}}, \quad \ddot{x}_{2}+2 n \dot{x}_{1}=\frac{\partial U}{\partial x_{2}}, \quad \ddot{x}_{3}=\frac{\partial U}{\partial x_{3}}
$$

where

$$
\begin{aligned}
& U=D\left[\pi \rho_{1}\left\{I-A_{1}\left(x_{1}^{2}+x_{2}^{2}\right)-A_{2} x_{3}^{2}\right\}+\frac{\mu}{\left[\left(1-x_{1}\right)^{2}+x_{2}^{2}+x_{3}^{2}\right]^{1 / 2}}\right. \\
& \left.+\frac{\mu \alpha_{2}}{\left[\left(1-x_{1}\right)^{2}+x_{2}^{2}+x_{3}^{2}\right]^{3 / 2}}-\frac{3 \mu \alpha_{2} x_{3}^{2}}{2\left[\left(1-x_{1}\right)^{2}+x_{2}^{2}+x_{3}^{2}\right]^{5 / 2}}+\frac{n^{2}\left\{\left(x_{1}-\mu\right)^{2}+x_{2}^{2}\right\}}{2}\right], \\
& n^{2}=\left(1+\frac{3}{2} \alpha_{1}+\frac{3}{2} \alpha_{2}\right) \text {. }
\end{aligned}
$$

These above equations of motion of the infinitesimal mass $m_{3}$ under the framework of the Robe's circular restricted three-body problem have been obtained by taking into account the shapes of the primaries, the full buoyancy force, the forces due to the gravitational attraction of the second primary, and the gravitational force exerted by the fluid of density $\rho_{1}$. In the case when the second primary is not an oblate spheroid (i.e., $\alpha_{2}=0$ ), the equations are the same as those of Hallan and Mangang [4].

\section{Position of Equilibrium Points}

The equilibrium points are the solutions of the equations:

$$
U_{x_{1}}=U_{x_{2}}=U_{x_{3}}=0
$$


That is,

$$
\begin{aligned}
U_{x_{1}}=D & {\left[-2 \pi \rho_{1} x_{1} A_{1}+\frac{\mu\left(1-x_{1}\right)}{\left[\left(1-x_{1}\right)^{2}+x_{2}^{2}+x_{3}^{2}\right]^{3 / 2}}\right.} \\
& \left.+\frac{3 \mu \alpha_{2}\left(1-x_{1}\right)}{2\left[\left(1-x_{1}\right)^{2}+x_{2}^{2}+x_{3}^{2}\right]^{5 / 2}}-\frac{15 \mu \alpha_{2}\left(1-x_{1}\right) x_{3}^{2}}{2\left[\left(1-x_{1}\right)^{2}+x_{2}^{2}+x_{3}^{2}\right]^{7 / 2}}+n^{2}\left(x_{1}-\mu\right)\right]=0, \\
U_{x_{2}=D x_{2}} & {\left[-2 \pi \rho_{1} A_{1}-\frac{\mu}{\left[\left(1-x_{1}\right)^{2}+x_{2}^{2}+x_{3}^{2}\right]^{3 / 2}}\right] } \\
& \left.-\frac{3 \mu \alpha_{2}}{2\left[\left(1-x_{1}\right)^{2}+x_{2}^{2}+x_{3}^{2}\right]^{5 / 2}}+\frac{15 \mu \alpha_{2} x_{3}^{2}}{2\left[\left(1-x_{1}\right)^{2}+x_{2}^{2}+x_{3}^{2}\right]^{7 / 2}}+n^{2}\right]=0, \\
U_{x_{3}=D x_{3}} & {\left[-2 \pi \rho_{1} A_{2}-\frac{\mu}{\left[\left(1-x_{1}\right)^{2}+x_{2}^{2}+x_{3}^{2}\right]^{3 / 2}}-\frac{2\left[\left(1-x_{1}\right)^{2}+x_{2}^{2}+x_{3}^{2}\right]^{5 / 2}}{3 \mu \alpha_{2}}\right] } \\
& \left.-\frac{15 \mu \alpha_{2} x_{3}^{2}}{\left[\left(1-x_{1}\right)^{2}+x_{2}^{2}+x_{3}^{2}\right]^{5 / 2}}+\frac{2\left[\left(1-x_{1}\right)^{2}+x_{2}^{2}+x_{3}^{2}\right]^{7 / 2}}{2}\right]=0 .
\end{aligned}
$$

\subsection{Equilibrium Points Near the Centre of the First Primary}

The positions of the equilibrium points near the first primary are the solutions of (3.2) when $U_{x_{1}}=0, x_{1} \neq 0, x_{2}=x_{3}=0, D \neq 0$, and $n^{2}=1+(3 / 2)\left(\alpha_{1}+\alpha_{2}\right)$. The $x_{1}$ coordinate of the equilibrium points are then the roots of the equation:

$$
-2 \pi \rho_{1} x_{1} A_{1}+\frac{\mu\left(1-x_{1}\right)}{\left|1-x_{1}\right|^{3}}+\frac{3 \mu \alpha_{2}\left(1-x_{1}\right)}{2\left|1-x_{1}\right|^{5}}+\left(1+\frac{3}{2} \alpha_{1}+\frac{3}{2} \alpha_{2}\right)\left(x_{1}-\mu\right)=0 .
$$

We first determine the roots of (3.5) in the absence of oblateness, that is, the case when the primaries are spherical. In this case, the roots are [4]

$$
x_{11}=1+\frac{\mu+\sqrt{\mu^{2}+8 \mu \pi \rho_{1} A_{1}-4 \mu}}{2\left(1-2 \pi \rho_{1} A_{1}\right)}, \quad x_{12}=1+\frac{\mu-\sqrt{\mu^{2}+8 \mu \pi \rho_{1} A_{1}-4 \mu}}{2\left(1-2 \pi \rho_{1} A_{1}\right)} .
$$

The term $A_{1}$ which appears in (3.2) and is due to the fluid mass affects these roots. Therefore, these roots will be real if the discriminant is nonnegative, that is if

$$
\mu+8 \pi \rho_{1} A_{1}-4 \geq 0 .
$$


When $(1 / 4) \mu \geq 1-2 \pi \rho_{1} A_{1}>0$, both roots are greater than unity and we reject them because they lie outside the first primary. Now, if $1-2 \pi \rho_{1} A_{1}<0$, we have $x_{12}>1$ and $x_{11}<1$. Further, we see that $x_{11}>-1$ when $1-2 \pi \rho_{1} A_{1}<-(3 / 4) \mu$. Thus, in the case when $1-2 \pi \rho_{1} A_{1}<-(3 / 4) \mu$, the point $\left(x_{11}, 0,0\right)$ lies within the first primary if $\left|x_{11}\right|<a_{1}$. When $1-2 \pi \rho_{1} A_{1}<-(3 / 4) \mu$, $\left|x_{11}\right|<a_{1} ; x_{11}$ is a root of (3.5). Hence for $1-2 \pi \rho_{1} A_{1}=0$, the only root is $x_{11}=2$ which lies outside the first primary and we neglect it. Hence, for $\alpha_{1}=0, \alpha_{2}=0, x_{11}=0$ is always a root of (3.5) and $x_{1}=x_{11}$ is also a root provided $1-2 \pi \rho_{1} A_{1}<-(3 / 4) \mu,\left|x_{11}\right|<a_{1}$.

Now, we find the roots of (3.5) when oblateness of both primaries is considered (i.e., $\left.\alpha_{1} \neq 0, \alpha_{2} \neq 0\right)$.

Let the roots be such that

$$
\begin{aligned}
& x_{1}=0+p_{1}, \quad\left|p_{1}\right| \ll 1, \\
& x_{1}=x_{11}+p_{2}, \quad\left|p_{2}\right| \ll 1 .
\end{aligned}
$$

Putting these values in (3.5), multiplying throughout by $\left(1-p_{1}\right)^{4}$, expanding and neglecting second and higher powers of $p_{1}, \alpha_{1}, \alpha_{2}$, as they are very small quantities, we have

$$
p_{1} \cong-\frac{3 \alpha_{1}}{2} \frac{\mu}{2 \pi \rho_{1} A_{1}-(1+2 \mu)} .
$$

Similarly, putting $x_{1}=x_{11}+p_{2}$ in (3.5) and then simplifying it, we get

$$
\begin{aligned}
& \left(1-x_{11}\right)^{4}\left[\left(x_{11}-\mu\right)\left(1+\frac{3}{2} \alpha_{1}+\frac{3}{2} \alpha_{2}\right)-2 \pi \rho_{1} A_{1} x_{11}\right] \\
& \quad+p_{2}\left(1-x_{11}\right)^{3}\left[\left(1-3 x_{11}\right)\left(1-2 \pi \rho_{1} A_{1}\right)+2 \mu\right] \\
& \quad+\mu\left(1-x_{11}\right)^{2}-2 p_{2}\left(1-x_{11}\right)\left[\left(1-x_{11}\right)^{2}\left\{x_{11}-2 \pi \rho_{1} A_{1} x_{11}-\mu\right\}+\mu\right]=-\frac{3 \mu \alpha_{2}}{2} .
\end{aligned}
$$

Multiplying (3.8) by $\left(1-x_{11}\right)^{2}$, simplifying and then using it in (3.10), we get

$$
p_{2} \cong-\frac{\left(1-x_{11}\right)\left[\left(x_{11}-\mu\right)\left((3 / 2) \alpha_{1}+(3 / 2) \alpha_{2}\right)\right]}{\left[\left(1-3 x_{11}\right)\left(1-2 \pi \rho_{1} A_{1}\right)+2 \mu\right]}-\frac{3 \mu \alpha_{2}}{2\left(1-x_{11}\right)^{3}\left[\left(1-3 x_{11}\right)\left(1-2 \pi \rho_{1} A_{1}\right)+2 \mu\right]}
$$

A substitution of (3.11) in the second equation of (3.8) at once gives the position of the other equilibrium point near the center of the first primary. 


\subsubsection{Positions of Circular Points}

The positions of the circular points are sought using the first two equations of system (3.1) with the conditions $x_{1} \neq 0, x_{2} \neq 0, x_{3}=0$; that is, they are the solutions of

$$
\begin{array}{r}
x_{1}\left[-2 \pi \rho_{1} A_{1}-\frac{\mu}{\left\{\left(1-x_{1}\right)^{2}+x_{2}^{2}\right\}^{3 / 2}}-\frac{3 \mu \alpha_{2}}{2\left\{\left(1-x_{1}\right)^{2}+x_{2}^{2}\right\}^{5 / 2}}+1+\frac{3}{2} \alpha_{1}+\frac{3}{2} \alpha_{2}\right] \\
+\frac{\mu}{\left\{\left(1-x_{1}\right)^{2}+x_{2}^{2}\right\}^{3 / 2}}+\frac{3 \mu \alpha_{2}}{2\left\{\left(1-x_{1}\right)^{2}+x_{2}^{2}\right\}^{5 / 2}}-\mu\left(1+\frac{3}{2} \alpha_{1}+\frac{3}{2} \alpha_{2}\right)=0, \\
-2 \pi \rho_{1} A_{1}-\frac{\mu}{\left\{\left(1-x_{1}\right)^{2}+x_{2}^{2}\right\}^{3 / 2}}-\frac{3 \mu \alpha_{2}}{2\left\{\left(1-x_{1}\right)^{2}+x_{2}^{2}\right\}^{5 / 2}}+1+\frac{3}{2} \alpha_{1}+\frac{3}{2} \alpha_{2}=0 .
\end{array}
$$

Solving the above equations and knowing that $\mu \neq 0$, we get

$$
\frac{1}{\left\{\left(1-x_{1}\right)^{2}+x_{2}^{2}\right\}^{3 / 2}}+\frac{3 \alpha_{2}}{2\left\{\left(1-x_{1}\right)^{2}+x_{2}^{2}\right\}^{5 / 2}}-n^{2}=0
$$

We let

$$
\left(1-x_{1}\right)^{2}+x_{2}^{2}=r^{2}
$$

Substituting (3.15) in (3.14), and simplifying, we get

$$
n^{2} r^{5}-r^{2}-\frac{3}{2} \alpha_{2}=0
$$

Now, we let

$$
r=1+\varepsilon, \quad \varepsilon \ll 1
$$

Substituting (3.16) in (3.15), neglecting second and higher powers of $\varepsilon$, we get

$$
\varepsilon=-\frac{1}{2} \alpha_{1}
$$

Therefore, (3.17) is now expressed as

$$
r \cong 1-\frac{1}{2} \alpha_{1} .
$$


A substitution of (3.14) in (3.13) yields

$$
2 \pi \rho_{1} A_{1}=n^{2}(1-\mu) .
$$

Therefore, when $2 \pi \rho_{1} A_{1}=n^{2}(1-\mu)$, the points on the circle given by (3.15) with $x_{3}=0$ and $r=1-(1 / 2) \alpha_{1}$ lying within the first primary are also equilibrium points. The general coordinates of these circular points are given by $(1+r \cos \theta, r \sin \theta, 0)$, where $\theta$ is a parameter. When $y=0$, the circular points coalesce to those lying on the line joining the primaries.

\subsubsection{Positions of Out-of-Plane Equilibrium Points}

The out-of-plane points have no analogy in the classical restricted three-body problem. However the investigation concerning these points in the photogravitational restricted threebody problem was first carried out by Radzievskii [10]. Afterwards, other researchers, for instance Douskos and Markellos [8], Singh and Leke [11], and so forth, have worked on the out-of-plane points. In this section, we locate these points for our study, as it has remained an open problem to date.

The positions of the out-of-plane equilibrium points of the Robe's problem with oblate primaries are the solutions of the first and last equations of (3.1) with $x_{2}=0, D \neq 0$; that is,

$$
\begin{gathered}
x_{1}\left[-2 \pi \rho_{1} A_{1}-\frac{\mu}{\left[\left(1-x_{1}\right)^{2}+x_{3}^{2}\right]^{3 / 2}}-\frac{3 \mu \alpha_{2}}{2\left[\left(1-x_{1}\right)^{2}+x_{3}^{2}\right]^{5 / 2}}+\frac{15 \mu \alpha_{2} x_{3}^{2}}{2\left[\left(1-x_{1}\right)^{2}+x_{3}^{2}\right]^{7 / 2}}+n^{2}\right] \\
+\frac{\mu}{\left[\left(1-x_{1}\right)^{2}+x_{3}^{2}\right]^{3 / 2}}+\frac{3 \mu \alpha_{2}}{2\left[\left(1-x_{1}\right)^{2}+x_{3}^{2}\right]^{5 / 2}}-\frac{15 \mu \alpha_{2} x_{3}^{2}}{2\left[\left(1-x_{1}\right)^{2}+x_{3}^{2}\right]^{7 / 2}}-n^{2} \mu=0 \\
x_{3}\left[-2 \pi \rho_{1} A_{2}-\frac{\mu}{\left[\left(1-x_{1}\right)^{2}+x_{3}^{2}\right]^{3 / 2}}-\frac{9 \mu \alpha_{2}}{2\left[\left(1-x_{1}\right)^{2}+x_{3}^{2}\right]^{5 / 2}}+\frac{15 \mu \alpha_{2} x_{3}^{2}}{2\left[\left(1-x_{1}\right)^{2}+x_{3}^{2}\right]^{7 / 2}}\right]=0 .
\end{gathered}
$$

From (3.22), since $x_{3} \neq 0$, we have

$$
-2 \pi \rho_{1} A_{2}=\frac{\mu}{\left[\left(1-x_{1}\right)^{2}+x_{3}^{2}\right]^{3 / 2}}+\frac{9 \mu \alpha_{2}}{2\left[\left(1-x_{1}\right)^{2}+x_{3}^{2}\right]^{5 / 2}}-\frac{15 \mu \alpha_{2} x_{3}^{2}}{2\left[\left(1-x_{1}\right)^{2}+x_{3}^{2}\right]^{7 / 2}} .
$$

Let

$$
l^{2}=\left(1-x_{1}\right)^{2}+x_{3}^{2} .
$$


Then, (3.23) and (3.21) may be written respectively:

$$
\begin{gathered}
\frac{15 \mu \alpha_{2} x_{3}^{2}}{2 l^{7}}=2 \pi \rho_{1} A_{2}+\frac{\mu}{l^{3}}+\frac{9 \mu \alpha_{2}}{2 l^{5}} \\
x_{1}\left[-2 \pi \rho_{1} A_{1}-\frac{\mu}{l^{3}}-\frac{3 \mu \alpha_{2}}{2 l^{5}}+\frac{15 \mu \alpha_{2} x_{3}^{2}}{2 l^{7}}+n^{2}\right]+\frac{\mu}{l^{3}}+\frac{3 \mu \alpha_{2}}{2 l^{5}}-\frac{15 \mu \alpha_{2} x_{3}^{2}}{2 l^{7}}-n^{2} \mu=0 .
\end{gathered}
$$

Now, from first equations (3.25), we get

$$
x_{3}= \pm \frac{l}{\sqrt{15 \mu \alpha_{2}}}\left[\mu\left(9 \alpha_{2}+2 l^{2}\right)+4 \pi \rho_{1} A_{2} l^{5}\right]^{1 / 2} .
$$

The use of (3.24) in second equation of (3.25) yields

$$
x_{1}=\frac{\left(2 \pi \rho_{1} A_{2}+n^{2} \mu\right) l^{5}+3 \mu \alpha_{2}}{\left[2 \pi \rho_{1}\left(A_{2}-A_{1}\right)+n^{2}\right] l^{5}+3 \mu \alpha_{2}} .
$$

We use the software package Mathematica (Wolfram 2004) to compute the coordinates of the out-of-plane equilibrium points denoted by $L_{6}$ and $L_{7}$ starting with the initial values $x_{1}=1-\mu$ and $x_{3}=\sqrt{3} \sqrt{\alpha_{2}}$ in the case where we have kept up to first order terms in both the numerator and the denominator; we then get

$$
\begin{aligned}
x_{1}= & \frac{2 \mu+3 \mu \alpha_{1}+4 A_{2} \pi \rho_{1}}{2+3 \alpha_{1}-4 A_{1} \pi \rho_{1}+4 A_{2} \pi \rho_{1}} \\
& -\frac{3\left\{\mu A_{1}+(1-\mu) A_{2}\right\} \alpha_{2} \pi \rho_{1}}{\left[1+3 \alpha_{1}\left(1+2 A_{2} \pi \rho_{1}-2 A_{1} \pi \rho_{1}\right)+4 \pi \rho_{1}\left(A_{2}-A_{1}+A_{1}^{2} \pi \rho_{1}+A_{2}^{2} \pi \rho_{1}-2 A_{1} A_{2} \pi \rho_{1}\right)\right]}, \\
x_{3}= & \frac{\sqrt{2 / 15} \mu \sqrt{\mu^{3}\left(1+2 \pi A_{2} \mu^{2} \rho_{1}\right)}}{\sqrt{\mu \alpha_{2}}}+\frac{7 \sqrt{3 \mu / 10}\left(\mu+2 \pi A_{2} \mu^{3} \rho_{1}\right) \sqrt{\alpha_{2}}}{2 \sqrt{\mu^{3}\left(1+2 \pi A_{2} \mu^{2} \rho_{1}\right)}} .
\end{aligned}
$$

The location of the out-of-plane equilibrium points can be obtained by solving numerically equations (3.26) and (3.27) using (3.24).

Now, from the expression for the density parameter

$$
D=\left(1-\frac{\rho_{1}}{\rho_{3}}\right)
$$

We assume that $\rho_{1} \neq \rho_{3}$, then $D>$ or $<0$. In the case when the density parameter is positive, we have

$$
\rho_{1}<\rho_{3} .
$$


Hence, numerically we choose

$$
a_{1}^{2}=0.94, \quad a_{2}^{2}=0.9, \quad a_{3}^{2}=0.82, \quad a_{4}^{2}=0.8, \quad \mu=0.01, \quad \pi=3.14 .
$$

Then,

$$
\alpha_{1}=0.024, \quad \alpha_{2}=0.02, \quad \rho_{1}=0.236
$$

Now, we perform a numerical exploration of computing the out-of-plane points in the case of the Earth-Moon system. To do this, we arbitrarily choose values for the $A_{i}(i=1,2)$. We found that when $A_{1}=2.5076$ and $A_{2}=2.555$, the positions of the out-of-plane points $\left(x_{1}, 0, \pm x_{3}\right)$ :

$$
x_{1}=3.3527, \quad x_{3}=0.271418 .
$$

The abscissae of the out-of-plane point is outside the possible region of motion of the infinitesimal mass and so we neglect it. However, in the case when the $A_{i}(i=1,2)$ are chosen such that

$$
\left|A_{1}-A_{2}\right| \ll 1, \quad A_{1}=0.7, A_{2}=0.68 \text { (say), }
$$

$A_{i} \in(0,0.7]$, the point $L_{6}$, and $L_{7}$ are, respectively,

$$
x_{1}=0.98611, \quad x_{3}=0.271381
$$

and lies within the fluid.

\section{Linear Stability of the Equilibrium Points}

In order to study the linear stability of any equilibrium point $\left(x_{10}, x_{20}, x_{30}\right)$ of an infinitesimal body, we displace it to the position $\left(x_{1}, x_{2}, x_{3}\right)$ such that

$$
\left(x_{10}+\xi, x_{20}+\eta, x_{30}+\zeta\right)
$$

where $\xi, \eta, \zeta$ are small displacements, and then linearize equation (2.3) to obtain the equations:

$$
\begin{gathered}
\ddot{\xi}-2 n \dot{\eta}=\left(U_{x_{1} x_{1}}^{0}\right) \xi+\left(U_{x_{1} x_{2}}^{0}\right) \eta+\left(U_{x_{1} x_{3}}^{0}\right) \zeta, \\
\ddot{\eta}+2 n \dot{\xi}=\left(U_{x_{1} x_{2}}^{0}\right) \xi+\left(U_{x_{2} x_{2}}^{0}\right) \eta+\left(U_{x_{2} x_{3}}^{0}\right) \zeta, \\
\ddot{\zeta}=\left(U_{x_{1} x_{3}}^{0}\right) \xi+\left(U_{x_{2} x_{3}}^{0}\right) \eta+\left(U_{x_{3} x_{3}}^{0}\right) \zeta,
\end{gathered}
$$

where the partial derivatives are evaluated at the equilibrium points. 


\subsection{Equilibrium Points Near the Center of the First Primary}

In order to consider the motion near any equilibrium point in the $x_{1} x_{2}$-plane, we let solutions of the first two equations of (4.2) be

$$
\xi=A \exp (\lambda t), \quad \eta=B \exp (\lambda t),
$$

where $A, B$, and $\lambda$ are constants.

Taking first and second derivatives of the above, substituting them into the first two equations of system (4.2) and has a non-zero solution when

$$
\left|\begin{array}{cc}
\left(\lambda^{2}-U_{x_{1} x_{1}}^{0}\right) & \left(2 n \lambda+U_{x_{1} x_{2}}^{0}\right) \\
\left(2 n \lambda-U_{x_{1} x_{2}}^{0}\right) & \left(\lambda^{2}-U_{x_{2} x_{2}}^{0}\right)
\end{array}\right|=0 .
$$

Expanding the determinant, we have

$$
\lambda^{4}-\left(U_{x_{1} x_{1}}^{0}+U_{x_{2} x_{2}}^{0}-4 n^{2}\right) \lambda^{2}+U_{x_{1} x_{1}}^{0} U_{x_{2} x_{2}}^{0}-\left(U_{x_{1} x_{2}}^{0}\right)^{2}=0
$$

Equation (4.5) is the characteristic equation corresponding to the variational equations (4.2) in the case when motion is considered in the $x_{1}, x_{2}$-plane.

Now, the values of the second-order partial derivatives of the equilibrium point $\left(x_{L}, 0,0\right)$, where $x_{L}=p_{1}$ stands for the first equilibrium and $x_{L}=x_{11}+p_{2}$ for the second one, are given as

$$
\begin{aligned}
& U_{x_{1} x_{1}}^{0}=D \mu\left[\frac{-\left(1-x_{L}\right)^{3}-\left(3 \alpha_{2} / 2\right)\left(1-x_{L}\right)+2 x_{L}\left(1-x_{L}\right)^{2}+6 x_{L} \alpha_{2}+n^{2}\left(1-x_{L}\right)^{5}}{x_{L}\left(1-x_{L}\right)^{5}}\right], \\
& U_{x_{2} x_{2}}^{0}=D \mu\left[\frac{-\left(1-x_{L}\right)^{3}-(3 / 2) \alpha_{2}\left(1-x_{L}\right)-x_{L}\left(1-x_{L}\right)^{2}-(3 / 2) \alpha_{2} x_{L}+n^{2}\left(1-x_{L}\right)^{5}}{x_{L}\left(1-x_{L}\right)^{5}}\right], \\
& U_{x_{3} x_{3}}^{0}=-D\left[2 \pi \rho_{1} A_{2}+\frac{\mu}{\left(1-x_{L}\right)^{3}}+\frac{9 \mu \alpha_{2}}{2\left(1-x_{L}\right)^{5}}\right], \quad U_{x_{1} x_{2}}^{0}=0=U_{x_{2} x_{3}}^{0}=U_{x_{1} x_{3}}^{0} .
\end{aligned}
$$

Substituting these in (4.2), we at once have the variational equations:

$$
\begin{gathered}
\ddot{\xi}-2 n \dot{\eta}=U_{x_{1} x_{1}}^{0} \xi, \\
\ddot{\eta}+2 n \dot{\xi}=U_{x_{2} x_{2}}^{0} \eta, \\
\ddot{\zeta}=-D\left[\frac{\mu}{\left(1-x_{L}\right)^{3}}+\frac{9 \mu \alpha_{2}}{2\left(1-x_{L}\right)^{5}}+2 \pi \rho_{1} A_{2}\right] \zeta,
\end{gathered}
$$

where the partial derivatives have been computed at each equilibrium point $x_{L}$. 
Now, (4.8) is independent of (4.7), the solution being a periodic function is bounded and therefore, the motion of the infinitesimal body in the $x_{3}$ direction is stable.

Now, the characteristic equation of the equilibrium points $\left(x_{L}, 0,0\right)$ corresponding to the system (4.7) is

$$
\lambda^{4}-\left(U_{x_{1} x_{1}}^{0}+U_{x_{2} x_{2}}^{0}-4 n^{2}\right) \lambda^{2}+U_{x_{1} x_{1}}^{0} U_{x_{2} x_{2}}^{0}=0
$$

where

$$
\begin{aligned}
& U_{x_{1} x_{1}}^{0}=3 D \mu\left(\frac{\alpha_{1}}{2 x_{L}}\right), \\
& U_{x_{2} x_{2}}^{0}=3 D \mu\left(-1+\frac{\alpha_{1}}{2 x_{L}}\right) .
\end{aligned}
$$

These equations have been obtained using binomial expansion and ignoring terms with second and higher power in $p_{1}, p_{2}, \alpha_{2}$, and their product.

Now, let $\lambda_{1}^{2}$ and $\lambda_{2}^{2}$ be the roots of (4.9), then, the equilibrium point is stable if both the roots are real and negative. This means that their sum must be negative and their product must be positive. Hence, the points $\left(x_{L}, 0,0\right)$ will be stable if the following two conditions hold:

$$
\begin{gathered}
\lambda_{1}^{2}+\lambda_{2}^{2}=U_{x_{1} x_{1}}^{0}+U_{x_{2} x_{2}}^{0}-4 n^{2}<0, \\
\lambda_{1}^{2} \lambda_{2}^{2}=U_{x_{1} x_{1}}^{0} U_{x_{2} x_{2}}^{0}>0 .
\end{gathered}
$$

Now, in the case of the first equilibrium point $x_{L}=p_{1}$, if we suppose in (4.10) that $p_{1}<0$ then, $U_{x_{1} x_{1}}^{0}<0$ since $0<\mu<1, D>0,0<\alpha_{1} \ll 1$ and when $p_{1}>0$, we have $U_{x_{1} x_{1}}^{0}>0$.

Similarly, in (4.11), if we suppose $p_{1}<0$ then $U_{x_{2} x_{2}}^{0}<0$.

For the case $p_{1}>0$, we will have $U_{x_{2} x_{2}}^{0}>0$ when $\alpha_{1}>2\left|p_{1}\right|$ which is not possible, hence $U_{x_{2} x_{2}}^{0}<0$.

In the case $0<p_{1}<\alpha_{1} / 2, U_{x_{1} x_{1}}^{0}>0$, and $U_{x_{2} x_{2}}^{0}>0$.

Also, if $0<\alpha_{1} / 2<p_{1}$, we see that $U_{x_{1} x_{1}}^{0}>0$ and $U_{x_{2} x_{2}}^{0}<0$. Thus, for the case $p_{1}<0$, the equilibrium point is stable. For $0<p_{1}<\alpha_{1} / 2$, the equilibrium point is stable if the condition (4.12) holds. When $0<\alpha_{1} / 2<p_{1}$, the equilibrium point is unstable.

Next, for the other equilibrium point positioned at $x_{L}=x_{11}+p_{2}$, when $x_{11}>0$, then $x_{11}^{\prime}>0$ since $\left|p_{2}\right| \ll 1$ and the equilibrium point is stable if the conditions (4.12) and (4.13) are satisfied. If $x_{11}<0$ then $x_{11}^{\prime}<0$; it makes $U_{x_{1} x_{1}}^{0}<0, U_{x_{2} x_{2}}^{0}<0$. Therefore, when $x_{11}<0$, both the conditions (4.12) and (4.13) are fulfilled and the equilibrium point is stable. 


\subsection{Circular Points}

At circular points $(1+r \cos \theta, r \sin \theta, 0)$, the values of the second partial derivatives with the use of (3.14) and neglecting the product $\alpha_{1} \alpha_{2}$ are

$$
\begin{aligned}
& U_{x_{1} x_{1}}^{0}=3 D \mu \cos ^{2} \theta\left(n^{2}+\alpha_{2}\right), \\
& U_{x_{1} x_{2}}^{0}=3 D \mu \cos \theta \sin \theta\left(n^{2}+\alpha_{2}\right), \\
& U_{x_{2} x_{2}}^{0}=3 D \mu \sin ^{2} \theta\left(n^{2}+\alpha_{2}\right), \\
& U_{x_{3} x_{3}}^{0}=-D\left[2 \pi \rho_{1} A_{2}+\mu\left(n^{2}+3 \alpha_{2}\right)\right] .
\end{aligned}
$$

Substituting these values in the variational equations (4.2), we get

$$
\begin{gathered}
\ddot{\xi}-2 n \dot{\eta}=3 D \mu \cos ^{2} \theta\left(n^{2}+\alpha_{2}\right) \xi+3 D \mu \cos \theta \sin \theta\left(n^{2}+\alpha_{2}\right) \eta+(0) \zeta, \\
\ddot{\eta}+2 n \dot{\xi}=3 D \mu \cos \theta \sin \theta\left(n^{2}+\alpha_{2}\right) \xi+3 D \mu \sin ^{2} \theta\left(n^{2}+\alpha_{2}\right) \eta+(0) \zeta, \\
\ddot{\zeta}=-D\left[2 \pi \rho_{1} A_{2}+\mu\left(n^{2}+3 \alpha_{2}\right)\right] \zeta .
\end{gathered}
$$

Equation (4.16) is independent of (4.15), it shows that the motion of the infinitesimal mass along the $x_{3}$-direction is stable.

Now, a substitution of these partial derivatives in the characteristic equation (4.5) yields

$$
\lambda^{4}-\left[3 D \mu\left(n^{2}+\alpha_{2}\right)-4 n^{2}\right] \lambda^{2}=0
$$

Let $\lambda^{2}=\Lambda$ in (4.17) then, we have

$$
\Lambda\left[\Lambda-\left\{3 D \mu\left(n^{2}+\alpha_{2}\right)-4 n^{2}\right\}\right]=0 .
$$

Hence, either

$$
\Lambda=0 \quad \text { or } \quad \Lambda=3 D \mu\left(n^{2}+\alpha_{2}\right)-4 n^{2}
$$


which implies that

$$
\lambda=0 \text { twice, } \text { or } \quad \lambda= \pm\left[3 D \mu\left(n^{2}+\alpha_{2}\right)-4 n^{2}\right]^{1 / 2} \text {. }
$$

Therefore, (4.20) gives the roots of the characteristic equation (4.17). Hence we conclude that the circular points are unstable due to the presence of multiple roots.

\subsection{Out-of-Plane Points}

To determine the stability of the out-of-plane equilibrium points, we consider the following partial derivatives:

$$
\begin{aligned}
& U_{x_{1} x_{1}}=D\left[-2 \pi \rho_{1} A_{1}+\mu\left\{\frac{\left[2\left(1-x_{1}\right)^{2}-x_{3}^{2}\right]}{\left\{\left(1-x_{1}\right)^{2}+x_{3}^{2}\right\}^{5 / 2}}\right\}\right. \\
& \left.+\frac{3}{2} \mu \alpha_{2}\left\{\frac{\left[4\left(1-x_{1}\right)^{2}-x_{3}^{2}\right]}{\left\{\left(1-x_{1}\right)^{2}+x_{3}^{2}\right\}^{7 / 2}}\right\}-\frac{15}{2} \mu \alpha_{2} x_{3}^{2}\left\{\frac{\left[6\left(1-x_{1}\right)^{2}-x_{3}^{2}\right]}{\left\{\left(1-x_{1}\right)^{2}+x_{3}^{2}\right\}^{9 / 2}}\right\}+n^{2}\right] \text {, } \\
& U_{x_{1} x_{3}}=D\left[\frac{-3 \mu\left(1-x_{1}\right) x_{3}}{\left\{\left(1-x_{1}\right)^{2}+x_{3}^{2}\right\}^{5 / 2}}-\frac{15}{2} \frac{\mu \alpha_{2}\left(1-x_{1}\right) x_{3}}{\left\{\left(1-x_{1}\right)^{2}+x_{3}^{2}\right\}^{7 / 2}}\right. \\
& \left.-\frac{15 \mu \alpha_{2}\left(1-x_{1}\right)}{2} \frac{\left.2 x_{3}\left[\left(1-x_{1}\right)^{2}+x_{3}^{2}\right]-7 x_{3}^{3}\right]}{\left\{\left(1-x_{1}\right)^{2}+x_{3}^{2}\right\}^{9 / 2}}\right] \\
& U_{x_{2} x_{2}}=D\left[-2 \pi \rho_{1} A_{1}-\mu\left\{\frac{\left(1-x_{1}\right)^{2}+x_{3}^{2}}{\left\{\left(1-x_{1}\right)^{2}+x_{3}^{2}\right\}^{5 / 2}}\right\}\right. \\
& \left.-\frac{3 \mu \alpha_{2}}{2}\left\{\frac{\left(1-x_{1}\right)^{2}+x_{3}^{2}}{\left\{\left(1-x_{1}\right)^{2}+x_{3}^{2}\right\}^{7 / 2}}\right\}+\frac{15 \mu \alpha_{2} x_{3}^{2}}{2}\left\{\frac{\left(1-x_{1}\right)^{2}+x_{3}^{2}}{\left\{\left(1-x_{1}\right)^{2}+x_{3}^{2}\right\}^{9 / 2}}\right\}+n^{2}\right], \\
& U_{x_{3} x_{3}}=D\left[-2 \pi \rho_{1} A_{2}-\mu\left\{\frac{\left(1-x_{1}\right)^{2}-2 x_{3}^{2}}{\left\{\left(1-x_{1}\right)^{2}+x_{3}^{2}\right\}^{5 / 2}}\right\}\right. \\
& \left.-\frac{9 \mu \alpha_{2}}{2}\left\{\frac{\left(1-x_{1}\right)^{2}-4 x_{3}^{2}}{\left\{\left(1-x_{1}\right)^{2}+x_{3}^{2}\right\}^{7 / 2}}\right\}+\frac{15 \mu \alpha_{2}}{2}\left\{\frac{3 x_{3}^{2}\left[\left(1-x_{1}\right)^{2}+x_{3}^{2}\right]-7 x_{3}^{4}}{\left\{\left(1-x_{1}\right)^{2}+x_{3}^{2}\right\}^{9 / 2}}\right\}\right] \text {. }
\end{aligned}
$$


Since $x_{2}=0$, therefore the partial derivatives to be computed at the out-of-plane equilibrium points are

$$
\begin{aligned}
& U_{x_{1} x_{2}}^{0}=0=U_{x_{2} x_{1}}^{0}=U_{x_{2} x_{3}}^{0}=U_{x_{3} x_{2}}^{0} \\
& U_{x_{1} x_{1}}^{0}=D\left[-2 \pi \rho_{1} A_{1}-2 \pi \rho_{1} A_{2}+\frac{\mu}{l^{3}}-\frac{3 \mu x_{3}^{2}}{l^{5}}+\frac{3 \mu \alpha_{2}}{2 l^{5}}-\frac{9 \mu \alpha_{2}}{2 l^{5}}-\frac{45 \mu \alpha_{2} x_{3}^{2}}{l^{7}}+\frac{105 \mu \alpha_{2} x_{3}^{4}}{2 l^{9}}+n^{2}\right], \\
& U_{x_{2} x_{2}}^{0}=D\left[-2 \pi \rho_{1} A_{1}+2 \pi \rho_{1} A_{2}+\frac{3 \mu \alpha_{2}}{l^{5}}+n^{2}\right], \\
& U_{x_{1} x_{3}}^{0}=-3\left(1-x_{1}\right) x_{3} D\left[\frac{\mu}{l^{5}}+\frac{15 \mu \alpha_{2}}{2 l^{7}}-\frac{35 \mu \alpha_{2} x_{3}^{2}}{2 l^{9}}\right], \\
& U_{x_{3} x_{3}}^{0}=D\left[\frac{3 \mu x_{3}^{2}}{l^{5}}+\frac{75 \mu \alpha_{2} x_{3}^{2}}{2 l^{7}}-\frac{105 \mu \alpha_{2} x_{3}^{4}}{2 l^{9}}\right] .
\end{aligned}
$$

Now, we let,

$$
\begin{array}{ll}
U_{x_{1} x_{1}}^{0}=U_{11}, & U_{x_{2} x_{2}}^{0}=U_{22}, \\
U_{x_{1} x_{3}}^{0}=U_{13}, & U_{x_{3} x_{3}}^{0}=U_{33}, \\
U_{x_{1} x_{2}}^{0}=U_{12}, & U_{x_{2} x_{3}}^{0}=U_{23} .
\end{array}
$$

Using (4.23), the variational equation can be recast in the form:

$$
\begin{gathered}
\ddot{\xi}-2 n \dot{\eta}=U_{11} \xi+U_{13} \zeta, \\
\ddot{\eta}+2 n \dot{\xi}=U_{22} \eta+U_{23} \zeta, \\
\ddot{\zeta}=U_{13} \xi+U_{33} \zeta .
\end{gathered}
$$

In order to consider the motion of the out-of-plane points, we let solution of the system (4.24) be

$$
\xi=A \exp (\lambda t), \quad \eta=B \exp (\lambda t), \quad \zeta=C \exp (\lambda t),
$$

where $A, B, C$, and $\lambda$ are constants. $\xi, \eta$, and $\zeta$ are the small displacements in the coordinates of the infinitesimal body.

Now, the characteristic equation corresponding to the variational equations (4.24) in the case of the out-of-plane point may be expressed as

$$
\lambda^{6}-a_{1} \lambda^{4}+a_{2} \lambda^{2}+a_{3}=0,
$$


where the coefficients of the characteristic equation (4.26) are such that

$$
\begin{aligned}
& a_{1}=D\left[-4 \pi \rho_{1} A_{1}-2 \pi \rho_{1} A_{2}+2 n^{2}\right] \\
& \begin{aligned}
& a_{2}=\frac{1}{4 l^{16}}[D[-9 \mu^{2} D x_{3}^{2}\left(-1+x_{1}\right)^{2}\left[2 l^{4}+5 \alpha_{2}\left(3 l^{2}-7 x_{3}^{2}\right)\right] \\
&+3 l^{4} \mu x_{3}^{2}\left[2 l^{4}+5 \alpha_{2}\left(5 l^{2}-7 \mu x_{3}^{2}\right)\right] \\
& \times\left[3 D \mu \alpha_{2}+2 l^{5}\left\{(-4+D) n^{2}-2 D A_{1} \pi \rho_{1}+2 D A_{2} \pi \rho_{1}\right\}\right] \\
&+D\left[6 l^{4} \mu x_{3}^{2}+3 \alpha_{2}\left\{l^{4} \mu-25 l^{2}-35 \mu x_{3}^{4}\right\}+2 l^{9}\left(n^{2}-2 A_{1} \pi \rho_{1}+2 A_{2} \pi \rho_{1}\right)\right] \\
&\left.\left.\times\left[3 \alpha_{2}\left\{l^{4} \mu-2 l^{2}\left(15+l^{2}\right) x_{3}^{2}+35 \mu x_{3}^{4}\right\}+2 l^{6}\left(l^{3} n^{2}+\mu-2 l^{3} A_{1} \pi \rho_{1}-2 l^{3} A_{2} \pi \rho_{1}\right)\right]\right]\right] \\
& a_{3}=\frac{3 D^{3}}{4 l^{18}} \mu x_{3}^{2}\left[2 l^{4}+5 \alpha_{2}\left(5 l^{2}-7 \mu x_{3}^{2}\right)\right]\left[n^{2}+\frac{3 \mu \alpha_{2}}{2 l^{5}}-2 A_{1} \pi \rho_{1}+2 A_{2} \pi \rho_{1}\right] \\
& \times\left[3 \alpha_{2}\left\{l^{4} \mu-2 l^{2}\left(15+l^{2}\right) \mu x_{3}^{2}+35 \mu x_{3}^{4}\right\}+2 l^{6}\left(l^{3} n^{2}+\mu-2 l^{3} A_{1} \pi \rho_{1}-2 l^{3} A_{2} \pi \rho_{1}\right)\right],
\end{aligned}
\end{aligned}
$$

where $l^{2}=\left(1-x_{1}\right)^{2}+x_{3}^{2}$.

These computations have been done using the software package Mathematica.

For the stability analysis of the out-of-plane equilibrium point, we compute numerically the partial derivatives calculated at the out-of-plane points with the use of (3.28) and the following numerical values:

$$
\begin{aligned}
& \mu=0.01, \quad \pi=3.14, \quad \alpha_{1}=0.024, \quad \alpha_{2}=0.02 \\
& A_{1}=0.7, \quad A_{2}=0.68, \quad \rho_{1}=0.236, \quad D=0.2133 \text {. }
\end{aligned}
$$

Now, substituting the above values in the characteristic equation (4.27), we get

$$
\lambda^{6}-0.192437 \lambda^{4}+0.248034 \lambda^{2}-0.0000197624=0 .
$$

Its roots are:

$$
\begin{aligned}
& \lambda_{1,2}=-0.545066 \pm 0.448239 i, \\
& \lambda_{3,4}= \pm 0.00892642, \\
& \lambda_{5,6}=0.545066 \pm 0.448239 i .
\end{aligned}
$$

The positive root and the positive real part of the complex roots induce instability at the out-of-plane point. Hence, the motion of the infinitesimal mass around the out-of-plane equilibrium points is unstable for the specific numerical example given here. However, fuller discussion of their stability remains a theme for future research. 


\section{Discussion}

The equation of motion (2.3) is different from those of Hallan and Mangang [4] due to oblateness of the second primary. If we assume that the second primary is not oblate (i.e., $\alpha_{2}=0$ ), then these equations will fully coincide with those of Hallan and Mangang [4].

Equation (3.9) gives the equilibrium position of the point $\left(P_{1}, 0,0\right)$ near the center of the first primary and fully coincides with that of Hallan and Mangang [4]. It shows that the position of this equilibrium point does not depend on oblateness of the second primary, while the other equilibrium point $\left(x_{11}+P_{2}, 0,0\right)$ given by (3.11) is different from that of Hallan and Mangang [4] due to the appearance of oblateness of the second primary. When $2 \pi \rho_{1} A_{1}=$ $n^{2}(1-\mu)$, points on the circle $\left(1-x_{1}\right)^{2}+x_{2}^{2}=r^{2}, x_{3}=0$ lying within the first primary are also equilibrium points. These points are affected by oblateness of both primaries. Equations (3.28) give the positions of the out-of-plane points when only linear terms in oblateness of the second primary are retained. We have been able to show that the oblateness of the primaries allows the existence of the out-of-plane equilibrium points in the $x_{1} x_{3}$-plane within the first primary. These points have no analogy in the previous studies of the Robe's restricted threebody problem.

The linear stability analysis of the equilibrium solutions of the problem is investigated with the help of characteristic roots. The characteristic equation (4.9) in the case of the equilibrium point $x_{L}=p_{1}$ near the center of the first primary is the same as that of Hallan and Mangang [4], while that of the other point $x_{L}=x_{11}+p_{2}$ near the center differs from that of Hallan and Mangang [4] due to oblateness of the second primary. The characteristic equation of the circular case (4.17) also differs from that of Hallan and Mangang [4] due to oblateness of the second primary. The stability in the first approximation of this configuration shows that points near the centre of the first primary are conditionally stable; the circular points are unstable. This confirms the earlier results of Hallan and Rana [3], Hallan and Mangang [4]. A numerical exploration shows that the out-of-plane equilibrium points are also unstable. This outcome validates the earlier results of Douskos and Markellos [8] and Singh and Leke [11] that the points are unstable.

\section{Conclusion}

We have derived the equations of motion and established the positions of the equilibrium points of the infinitesimal body in the Robe's [1] restricted three-body problem with oblateness. The term "oblateness" is used in the sense that both primaries are considered as oblate spheroids under the effects of the full buoyancy force exerted by the fluid on the infinitesimal mass.

We have obtained one equilibrium point $\left(P_{1}, 0,0\right)$ near the centre of the first primary which will be on the left or right of the centre of the first primary accordingly as $2 \pi \rho_{1} A_{1}-$ $2 \mu><1$. This point is the same as that of Hallan and Mangang [4]. In addition to this, another equilibrium point $\left(x_{11}+P_{2}, 0,0\right)$ is found within the first primary on the line joining the center of the primaries when $1-2 \pi \rho_{1} A_{1}<-3 \mu / 4$ and $\left|x_{11}\right|<a_{1}$. When $2 \pi \rho_{1} A_{1}=n^{2}(1-\mu)$, points on the circle $\left(1-x_{1}\right)^{2}+x_{2}^{2}=r^{2}, x_{3}=0$ lying within the first primary are also equilibrium points. We call them circular points. Finally, we have been able to show that the oblateness of the primaries allows the existence of the out-of-plane equilibrium points in the $x_{1} x_{3}$-plane within the first primary. 
The result of this paper can be summarized as follows. The restricted three-body problem under the framework of the Robe's [1] problem with oblate primaries has the equilibrium points of the type: points near the center of the first primary, points on the circle (circular points), and two out-of-plane points $L_{6,7}$. It is seen that points near the first primary are conditionally stable, the circular points are unstable, while the out-of-plane equilibrium points are unstable for the specific numerical example given here. The effect of drag forces as considered by Giordano et al. [2] under the present context, particularly as regards the analysis of the properties of the equilibrium points located inside the first primary, will be interesting.

\section{References}

[1] H. A. G. Robe, "A new kind of three body problem," Celestial Mechanics and Dynamical Astronomy, vol. 16, pp. 343-351, 1977.

[2] C. M. Giordano, A. R. Plastino, and A. Plastino, "Robe's restricted three-body problem with drag," Celestial Mechanics and Dynamical Astronomy, vol. 66, no. 2, pp. 229-242, 1996.

[3] P. P. Hallan and N. Rana, "The existence and stability of equilibrium points in the Robe's restricted three-body problem," Celestial Mechanics \& Dynamical Astronomy, vol. 79, no. 2, pp. 145-155, 2001.

[4] P. P. Hallan and K. B. Mangang, "Existence and linear stability of equilibrium points in the Robe's restricted three body problem when the first primary is an oblate spheroid," Planetary and Space Science, vol. 55, no. 4, pp. 512-516, 2007.

[5] A. R. Plastino and A. Plastino, "Robe's restricted three-body problem revisited," Celestial Mechanics and Dynamical Astronomy, vol. 61, no. 2, pp. 197-206, 1995.

[6] R. K. Sharma and P. V. Subba Rao, "Stationary solutions and their characteristic exponents in the restricted three-body problem when the more massive primary is an oblate spheroid," Celestial Mechanics, vol. 13, no. 2, pp. 137-149, 1976.

[7] J. Singh and B. Ishwar, "Stability of triangular points in the generalized photogravitational restricted three-body problem," Bulletin of the Astronomical Society of India, vol. 27, pp. 415-424, 1999.

[8] C. N. Douskos and V. V. Markellos, "Out-of-plane equilibrium points in the restricted three-body problem with oblateness," Astronomy and Astrophysics, vol. 446, no. 1, pp. 357-360, 2006.

[9] J. Singh and O. Leke, "Stability of the photogravitational restricted three-body problem with variable masses," Astrophysics and Space Science, vol. 326, pp. 305-314, 2010.

[10] V. V. Radzievskii, "The restricted problem of three bodies taking account of light pressure," Astronomicheskii Zhurnal, vol. 27, pp. 250-256, 1950.

[11] J. Singh, "Combined effects of perturbations, radiation and oblateness on the nonlinear stability of triangular points in the restricted three-body problem," Astrophysics and SpaceScience, vol. 332, pp. 331-339, 2011. 


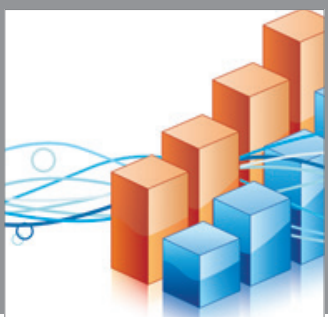

Advances in

Operations Research

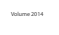

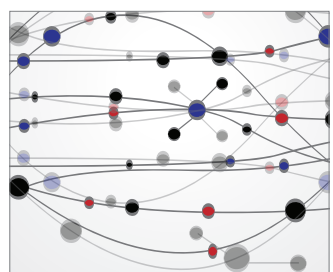

\section{The Scientific} World Journal
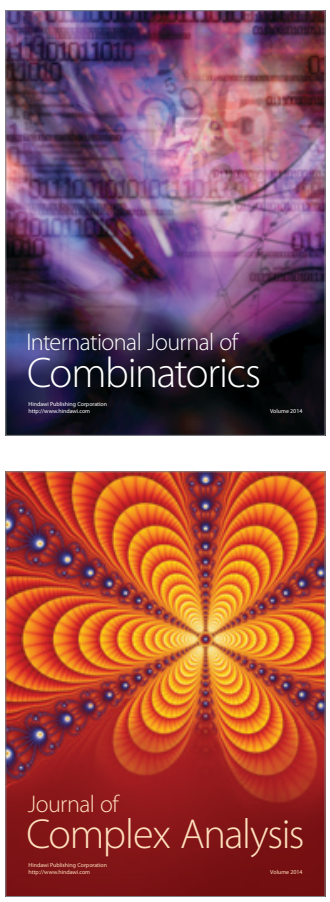

International Journal of

Mathematics and

Mathematical

Sciences
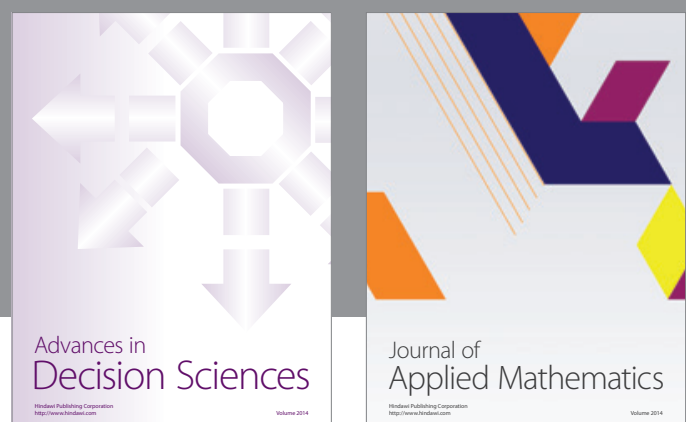

Journal of

Applied Mathematics
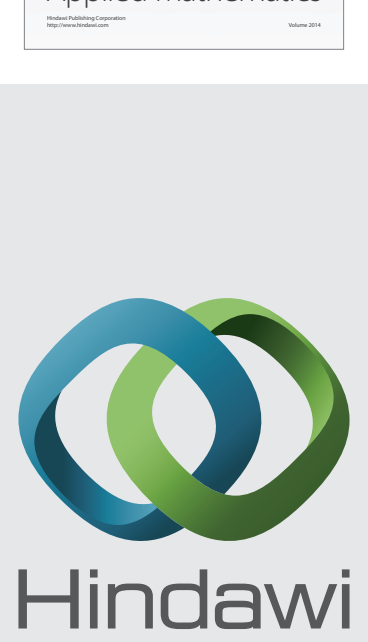

Submit your manuscripts at http://www.hindawi.com
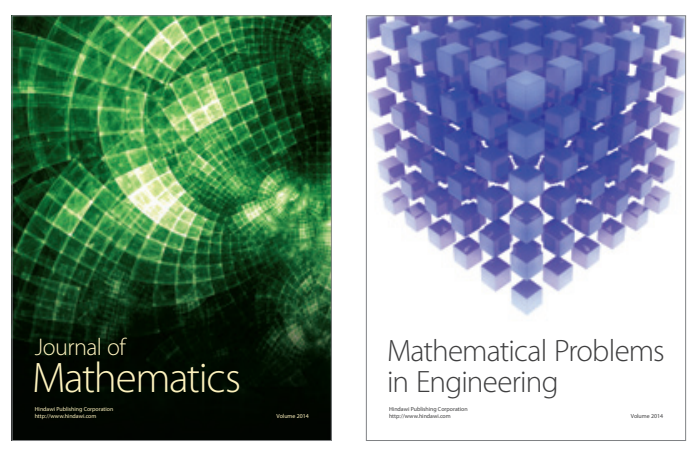

Mathematical Problems in Engineering
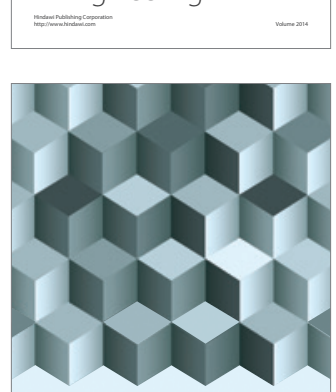

Journal of

Function Spaces
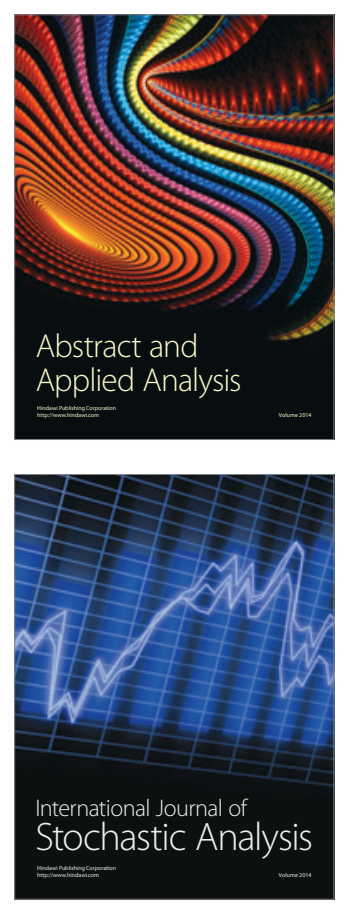

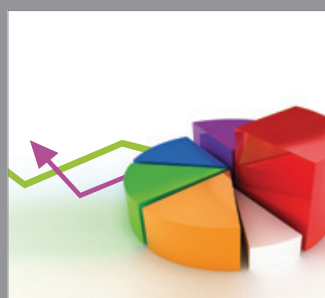

ournal of

Probability and Statistics

Promensencen
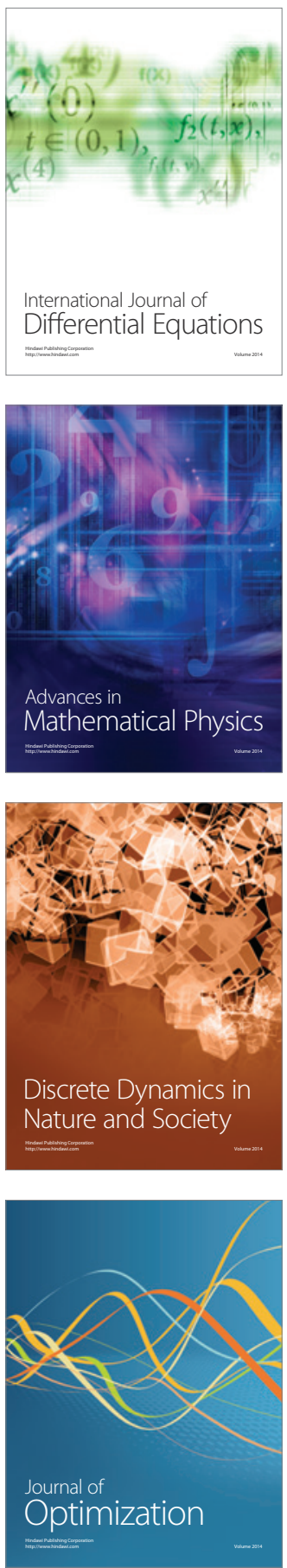